\title{
Prognostic Effects of Short-term Blood Pressure Variability Among Patients With Heart Failure With Reduced Ejection Fraction
}

\author{
Havyarimana Juvenal ${ }^{1}$ Chengkun Kou ${ }^{1}$ : Wang Ming Ming ${ }^{1}$ : Yu Jing ${ }^{1 *}$ \\ 1,2,3: Department of Crdiology, Lanzhou University Second Hospital, Lanzhou, Gansu, China \\ *Corresponding: Department of Crdiology, Lanzhou University Second Hospital, 82 \\ Cuiyingmen St, Lanzhou, 730030, Gansu,Peaple's Republic of China \\ Tel +86931519 0694, Fax +869315190694
}

doi: 10.51505/ijmshr.2022.6109

URL: http://dx.doi.org/10.51505/ijmshr.2022.6109

\begin{abstract}
Objective: To assess the prognostic impact of short-term blood pressure variability among heart failure patients with reduced ejection fraction.

Methods: The pooled endpoint of cardiac death, stroke, myocardial infarction, and hospitalization were examined in $156 \mathrm{HF}$ subjects with reduced EF. All subjects had their blood pressure measured ambulatorily. Mean average real variability (ARV) 24-hour systolic BP $(\mathrm{n}=78 ; \mathrm{n}=78)$ was used to classify individuals as having the lowest $(0.77 \mathrm{mmHg})$ or the highest (0.77 mmHg) ARV.
\end{abstract}

\section{Results:}

Throughout the follow-up period (7.6 \pm 3.6 months), 107 events occurred, of which $29(19 \%)$ were cardiac deaths, 18 (12\%) myocardial infarction, 9 (6\%) stroke, and 51(33\%) hospitalizations. Patients with low 24-hour systolic ARV had significantly higher cardiac death and MI than those with high 24-hour systolic ARV. Risk of negative outcomes identified as an independent variable by logistic regression analysis; age (AOR 1.08, 95\% CI: 1.01-1.15; p = 0.01 ), coronary artery disease (AOR $0.34,95 \% \mathrm{CI}: 0.12-0.98 ; \mathrm{P}=0.04$ ), valvular cardiomyopathy (AOR 0.08, 95\% CI: 0.09-0.82; $\mathrm{P}=0.03$ ), daytime systolic BP (AOR 1.25,95\% CI:1.04-1.49; $\mathrm{p}=0.01$ ) daytime diastolic BP (AOR 1.07, 95\% CI: 1.00-1.14; $\mathrm{p}=0.04$ ) and 24hour DBP (AOR 1.12, 95\% CI: 1.02-1.22; $\mathrm{P}=0.01$ )

\section{Conclusion:}

Low ARV of 24-hour systolic BP is associated with greater cardiovascular risk indicators daytime systolic BP variability, night-time systolic BP variability, 24-h systolic BP variability, and 24-h diastolic BP variability with $\mathrm{HF}$ with reduced EF patients.

Keywords: Average real variability, Blood pressure variability, Heart failure, Prognostic effect, Reduced ejection fraction. 


\section{International Journal of Medical Science and Health Research}

Vol. 6, No. 01; 2022

ISSN: $2581-3366$

\section{Introduction}

The variability of BP is a complicated process that increases when blood pressure levels fluctuate over time. Various periods (Parati, Ochoa, Lombardi, \& Bilo, 2013) highlight the physiological distinctions between the regulatory systems that activate different forms of BPV and offer the mean assessment of blood pressure (BP) level. Monitored blood pressure ambulatorily has recently been shown to be a stronger predictor of major cardiovascular events than clinic-based BP readings. Monitored blood pressure ambulatorily assists in lowering the number of probable erroneous readings while also providing insight into the dynamic fluctuation of blood pressure (Dadlani, Madan, \& Sawhney, 2019)

Higher systolic BP is linked to a lower death rate in people with chronic heart failure. Paradoxically, in hypertensive subjects, high systolic BP is an unfavorable risk factor, but low systolic blood pressure is always linked with a more favorable prognosis in hypertensive patients (Raphael et al., 2009; Regnault et al., 2014). This result can be explained partly because, in chronic heart failure (CHF), a higher blood pressure corresponds to higher cardiac output (Güder et al., 2009). In a meta-analysis, Raphael et al. highlighted a paradox in the impact of increased systolic BP on mortality in CHF patients, finding a $13 \%$ reduction in premature death with an increased systolic BP over $10 \mathrm{mmHg}$ (Raphael et al., 2009).

Over the past decades, the significant prognosis of each BP profiling determinant, such as systolic BP, diastolic BP, heart rate (HR), and BP variability (BPV), as well as standard deviation (SD), average real variability (ARV), has primarily been investigated in hypertensive patients (Clement et al., 2003; Coccina, Pierdomenico, Cuccurullo, \& Pierdomenico, 2019), but few in chronic HF patients. A high 24-hour systolic BP ARV is linked to a greater risk of cardiovascular disease in a hypertensive population (Coccina et al., 2019).

Mena et al. (L. Mena et al., 2005) recommended using the average veal variation (ARV) index to enhance the predictive potential of 24-hour BPV. This technique focuses on changes that occur in a short period, thus overcoming some of SD's constraints. It only illustrates the spread of blood pressure values around the mean. Thus, as a result, further studies may be devoted to understanding the prognostic effect of short-term BPV heart failure subjects with reduced ejection fraction. This research aimed to assess how short-term blood pressure fluctuation affected patients' prognosis in $\mathrm{HF}$ with reduced $\mathrm{EF}$.

\section{Methods}

\subsection{Subjects}

Subjects were collected retrospectively from the cardiology department's database, which comprised admitted patients for systolic HF at the cardiology ward of Lanzhou University Second Hospital between 2014 and 2020. Nevertheless, a single reading was required to determine the average real variability (ARV). Clinical assessments, standard laboratory testing, echocardiographic investigations, and non-invasive ambulatory blood pressure monitoring were undertaken on all patients. 


\section{International Journal of Medical Science and Health Research}

Vol. 6, No. 01; 2022

ISSN: $2581-3366$

The criteria for inclusion were as follows: $\geq 18$ years of aged or older of patients, of both genders, HFrEF event in life, ABPM at admission or one day after admission, and stable heart failure with $\leq 40 \%$ of EF in New York Heart Association functional classification (NYHA) IIIV. The criteria for exclusion were: patients <18 years old, with incomplete ambulatory blood pressure (ABP) recordings, infection, and dialysis. The local institutional ethics committee approved the research.

\subsection{Ambulatory blood pressure monitoring (ABPM)}

24-h monitoring of ambulatory blood pressure was undertaken as previously determined in chronic HF(Goyal, Macfadyen, Watson, \& Lip, 2005) using the oscillometer technique of the A\&D TM-2430 (Palatini et al., 1998). Blood pressure was taken every 15 minutes between 7:00 a.m. and 9:59 p.m. throughout the day and every 30 minutes between 10:00 p.m. and 6:59 a.m. during the night. The BP was measured in millimetres of mercury ( $\mathrm{mmHg}$ ).

A trained nurse fitted the ABPM devices on the right arms of the patients 24 hours after their admission. During the test, patients were instructed to relax their cuffed arms, after which they were given a dairy sheet each to help keep track of any unexpected events.

Diurnal (awake period), nocturnal (asleep period), and 24-hour systolic and diastolic blood pressures were assessed as ambulatory blood pressure parameters. As previously mentioned, we measured blood pressure variability using the 24-hour systolic BP of average real variability (ARV) (Coccina et al., 2019). The ARV 24-hour calculates the average of successive measures of the absolute differences and thereby adjusts the sequence in which the BP readings were taken. It is computed using the formula below (L. Mena et al., 2005):

$$
A R V=\frac{1}{N-1} \sum_{K-1}^{N-1}\left|B P_{K+1}-B P_{K}\right|
$$

$\mathrm{N}$ represents the valid number of BP readings, and $\mathrm{K}$ denotes the sequence of readings from each monitored participant.

In this present research, the effect of ARV on 24-hour SBP was investigated. Subjects with ARV 24-hour systolic BP less than or greater than the medium $(0.772 \mathrm{mmHg}$ or $0.772 \mathrm{mmHg})$ were identified as having the lowest or the highest $\mathrm{BP}$ variability, respectively. If systolic $\mathrm{BP}$, or diastolic BP, or pulse pressure was more 260 or less $70 \mathrm{mmHg}$, or was more 150 or less $40 \mathrm{mmHg}$, or more 150 or less $20 \mathrm{mmHg}$, respectively (Pierdomenico et al., 1995); automatic editing was performed on recordings (i.e. removed).

Patients had high-quality technical recordings (at least 20 valid measurements while awake with 2 measurements per hour, at least 7 valid measures were taken at night with 1 valid measurement per hour, and $70 \%$ of the 24 -hour predicted measurements must be valid). 


\section{International Journal of Medical Science and Health Research}

Vol. 6, No. 01; 2022

ISSN: $2581-3366$

\subsection{Follow-up of patients}

Patients were contacted by phone by the study's author during the follow-up phase or via interactions with family members. Subjects who did not provide new information after ABPM in the preceding month were judged missing for follow-up. During 15 months of follow-up, the occurrence outcomes consisted of a combination of cardiovascular events involving cardiac death and myocardial infarction, stroke, and hospitalization.

\subsection{Statistical analysis}

The mean and standard deviation were used to present continuous data. Percentages were used to express categorical data. The comparison of the two groups was made using an unpaired t-test and $\chi 2$-test. We analyzed the receiver operating characteristic (ROC) curve. The factors affecting the outcomes were studied using multiple linear regression. The sensitivity and specificity of the tested parameters were determined using ROC curves. SPSS 25 was used, and the value of $p$ $<0.05$ had statistical significance.

\section{Result}

Characteristics of baseline and blood pressure values of participants following 24-h systolic ARV are reported in Table 1. The allocated medicine diuretics beta-blockers were highly significant in patients with low 24-h systolic ARV. BMI and digoxin as allocated medicine were significantly low in patients with low 24-h systolic ARV. Ambulatory blood pressure monitoring (ABPM), daytime systolic BP, daytime diastolic BP, night-time systolic BP, night-time diastolic BP, 24-hour systolic BP, 24-h diastolic BP were significantly low in patients with low 24-h systolic ARV compared with those with high systolic ARV.

During the follow-up, of 156 subjects included,107 cardiovascular events occurred; among them $29(19 \%)$ of cardiac death, $18(12 \%)$ of myocardial infarction, $9(6 \%)$ of stoke, and $51(33 \%)$ of hospitalization for worsened heart failure specifically, with an average follow-up of $7.6 \pm 3.6$ months. There was not anyone who missed a follow-up.

The higher risk of having cardiovascular events in patients with the lowest and higheatest 24hours systolic average real variability is reported in Table 2. Myocardial infarction (MI) and Cardiac death were significantly related with blood pressure variability index in low 24-h systolic ARV ((adjusted odd ratio =AOR) AOR= 3.5 (1.1-11.6), $\mathrm{P}=0.03, \mathrm{AOR}=0.3$ (0.1-0.8), $\mathrm{P}=0.02 ; \mathrm{AOR}=3.5(1.1-11.6), \mathrm{P}=0.03))$ respectively.

According to multivariate logistic regression analysis, all unfavorable outcomes were independently related to age, medical history of coronary artery disease and valvular cardiomyopathy, daytime systolic BP, daytime diastolic BP, and 24-hour diastolic BP. The independent variables for negative outcomes were reported in Table 3.

After adjusting for variables related to poor prognosis in subjects with HFrE; (age, EF, daytime SBP, daytime DSP, daytime PP, night-time SBP, night-time DBP, night-time PP, 24-h SBP, 24-h DBP, and 24-h PP), Four independent factors were discovered to be very sensitive and specific for predicting poor outcomes in subjects with HFrE: daytime SBP (sensitivity $88 \%$, specificity 
Vol. 6, No. 01; 2022

ISSN: 2581-3366

59\%: under area curve $=0.794,95 \%$ CI: $0.659-0.928, \mathrm{p}=0.003$ ), nighttime SBP (sensitivity $88 \%$, specificity 74\%; area under curve $=0.746,95 \%$ CI: 0.577-0.915, $\mathrm{p}=0.013$ ), 24-h SBP (sensitivity $88 \%$, specificity $63 \%$; area under curve $=0.784,95 \%$ CI: $0.645-0.923, p=0.004$ ) and 24-h SBP (sensitivity $88 \%$, specificity $55 \%$; area under curve $=0.741,95 \%$ CI: 0.611 $0.872, \mathrm{p}=0.015$ ). The sensitivity and specificity of short-term BPV's prognostic impact as a predictive contributing factor for cardiovascular events were explored by a receiver operating characteristic (ROC) analysis. (Figure 1)

Table1: Baseline characteristics according to 24-hours systolic average real variability ARV

\begin{tabular}{|c|c|c|c|}
\hline \multirow{2}{*}{ Parameters } & \multirow{2}{*}{$\begin{array}{l}\text { Low 24hoursystolic } \\
\text { ARV n=78 }\end{array}$} & \multicolumn{2}{|c|}{ High 24-hour systolic } \\
\hline & & ARV $\mathrm{n}=78$ & $\mathrm{P}$ \\
\hline \multicolumn{4}{|l|}{ Gender, n (\%) } \\
\hline Men & $58(74.4)$ & $46(59)$ & 0.042 \\
\hline Female & $20(25.6)$ & $32(41)$ & \\
\hline Age, years Mean \pm SD & $58 \pm 14$ & $62 \pm 15$ & 0.822 \\
\hline BMI, kg/m2 Mean \pm SD & $24 \pm 3$ & $25 \pm 4$ & 0.019 \\
\hline Ejection Fraction, Mean \pm SD & $30 \pm 6$ & $31 \pm 6$ & 0.735 \\
\hline \multicolumn{4}{|l|}{ NYHA Class, n (\%) } \\
\hline II & $4(5.1)$ & $14(17.7)$ & 0.039 \\
\hline III & $26(33.3)$ & $20(25.6)$ & \\
\hline IV & $48(61.5)$ & $44(56.4)$ & \\
\hline \multicolumn{4}{|l|}{ Medical history } \\
\hline Hypertension $\mathrm{n}(\%)$ & $33(42.3)$ & $46(59)$ & 0.037 \\
\hline Smoker, n $(\%)$ & $13(16.7)$ & $8(10.3)$ & 0.241 \\
\hline Congestive heart failure, $\mathrm{n}(\%)$ & $14(17.9)$ & $21(26.9)$ & 0.179 \\
\hline Diabetes mellitus, $\mathrm{n}(\%)$ & $17(21.8)$ & $24(30.8)$ & 0.203 \\
\hline Valvular cardiomyopathy,n (\%) & $6(7.7)$ & $7(9)$ & 0.772 \\
\hline Dilated cardiomyopathy, n (\%) & $50(64.1)$ & $37(47.4)$ & 0.036 \\
\hline Atrial fibrillation, $\mathrm{n}(\%)$ & $23(29.5)$ & $20(25.6)$ & 0.591 \\
\hline Coronary artery disease, $\mathrm{n}(\%)$ & $18(23.1)$ & $27(34.6)$ & 0.112 \\
\hline \multicolumn{4}{|l|}{ Allocated treatment } \\
\hline Diuretics, n (\%) & $78(100)$ & $67(85.9)$ & 0.000 \\
\hline
\end{tabular}


Vol. 6, No. 01; 2022

ISSN: 2581-3366

CAEI, n (\%)

Beta-blocker

Aspirin, n (\%)

Statin, n (\%)

Digoxin, $\mathrm{n}(\%)$

ABPM

Daytime SBP, $\mathrm{mmHg}, \mathrm{M} \pm \mathrm{SD}$

Daytime DBP, $\mathrm{mmHg}, \mathrm{M} \pm \mathrm{SD}$

Daytime $\mathrm{PP}, \mathrm{mmHg}, \mathrm{M} \pm \mathrm{SD}$

Nighttime SBP, $\mathrm{mmHg}, \mathrm{M} \pm \mathrm{SD}$

Nighttime DBP, mmHg, $\mathrm{M} \pm \mathrm{SD}$

Nighttime PP, $\mathrm{mmHg}, \mathrm{M} \pm \mathrm{SD}$

24-h SBP, mmHg, $\mathrm{M} \pm$ SD

24-h DBP, mmHg, M \pm SD

24-h PP, mmHg, M \pm SD

Dipping, $\mathrm{mmHg}, \mathrm{M} \pm \mathrm{SD}$
$52(66.7)$

73 (93.6)

23 (29.5)

$25(32.1)$

23 (29.5)

$$
\begin{gathered}
110 \pm 16 \\
65 \pm 6 \\
74 \pm 10 \\
110 \pm 15 \\
66 \pm 11 \\
71 \pm 14 \\
110 \pm 7 \\
66 \pm 8 \\
73 \pm 11 \\
1 \pm .49
\end{gathered}
$$

$52(66.7)$

$62(79.5)$

35 (44.9)

27 (34.6)

14 (17.9)

$113 \pm 18$

$68 \pm 8$

$75 \pm 11$

$112 \pm 15$

$67 \pm 12$

$72 \pm 14$

$113 \pm 9$

$69 \pm 10$

$74 \pm 11$

$1 \pm .41$
1

0.01

0.047

0.734

0.090

0.008

0.006

0.414

0.027

$\mathbf{0 . 0 1 0}$

0.195

0.014

0.028

0.791

0.047

NYHA: New York Heart Association functional class, BMI: Body mass index, CAEI: Angiotensin-converting enzyme inhibitor, ARV: Average real variability, SBP: Systolic blood pressure, DBP: Diastolic blood pressure, PP: Pulse pressure, M: Mean, SD: Standard deviation.

Table 2: Risks of cardiovascular events (negative outcomes) for the low vs High 24-hours systolic average real variability

\begin{tabular}{lllllll}
\hline & $\begin{array}{l}\text { Participants } \\
\mathrm{N}=(156), \%\end{array}$ & LSARV & HSARV & AOR & 95\% CI & P-value \\
\hline Cardiac death & $29(18.5)$ & 16 & 13 & 0.35 & $0.15-0.84$ & $\mathbf{0 . 0 2}$ \\
MI & $18(11.5)$ & 11 & 7 & 3.58 & $1.10-11.64$ & $\mathbf{0 . 0 3}$ \\
Stoke & $9(5.7)$ & 7 & 2 & 0.17 & $0.03-1.00$ & 0.05 \\
Hospitalization & $51(32.6)$ & 27 & 24 & 0.89 & $0.4-1.8$ & 0.76 \\
\hline
\end{tabular}

MI: myocardial infarction, LSARV: Low systolic average real variability, HSARV: High systolic average real variability AOR: Adjusted odds ratio, CI: Confidence interval.

We adjusted the blood pressure variability index: daytime systolic blood pressure, daytime diastolic blood pressure, daytime pulse pressure, night-time systolic blood pressure, night-time diastolic blood pressure, night-time pulse pressure, 24-h systolic blood pressure, 24-h diastolic Blood pressure, 24-h pulse pressure. 
International Journal of Medical Science and Health Research

Vol. 6, No. 01; 2022

ISSN: 2581-3366

Table 3: Multiple logistic regression for poor outcomes and cardiac death

\begin{tabular}{|c|c|c|c|c|}
\hline \multirow[t]{2}{*}{ Parameters } & \multicolumn{2}{|l|}{ Negative outcomes } & \multicolumn{2}{|l|}{ Cardiac death } \\
\hline & OR $(95 \% \mathrm{CI})$ & $\mathrm{P}$ & OR $(95 \% \mathrm{CI})$ & $\mathrm{P}$ \\
\hline Gender & $1.33(0.33-5.37)$ & 0.68 & $0.41(0.13-1.25)$ & 0.11 \\
\hline Age & $1.08(1.01-1.15)$ & 0.01 & $0.99(0.95-1.04)$ & 0.92 \\
\hline Hypertension & $2.41(0.68-8.46)$ & 0.17 & $2.32(0.78-6.92)$ & 0.12 \\
\hline $\mathrm{EF}$ & $1.07(0.96-1.19)$ & 0.21 & $1.00(0.91-1.09)$ & 0.99 \\
\hline Smoking & $0.76(0.23-2.44)$ & 0.64 & $0.58(0.09-3.42)$ & 0.52 \\
\hline Body mass index & $1.06(0.87-1.31)$ & 0.52 & $0.98(0.85-1.11)$ & 0.76 \\
\hline Dilated cardiomyopathy & $1.45(0.52-4.04)$ & 0.47 & $0.43(0.11-1.71)$ & 0.23 \\
\hline Coronary artery disease & $0.34(0.12-0.98)$ & 0.04 & $0.41(0.09-1.84)$ & 0.24 \\
\hline Diabetes mellitus & $1.43(0.56-3.60)$ & 0.44 & $1.70(0.53-5.40)$ & 0.36 \\
\hline Congestive heart failure & $0.93(0.29-2.97)$ & 0.91 & $1.78(0.39-8.06)$ & 0.45 \\
\hline Valvular cardiomyopathy & $0.08(0.09-0.82)$ & $\mathbf{0 . 0 3}$ & $0.13(0.01-1.42)$ & 0.09 \\
\hline Beta-blocker & $1.11(0.92-1.85)$ & 0.93 & $0.78(0.17-3.51)$ & 0.75 \\
\hline Aspirin & $2.32(0.62-8.64)$ & 0.20 & $0.51(0.12-2.14)$ & 0.63 \\
\hline Diuretics & $0.31(0.03-2.69)$ & 0.29 & $0.79(0.19-3.19)$ & 0.74 \\
\hline ACEI & $2.46(0.60-9.99)$ & 0.20 & $1.72(0.42-6.95)$ & 0.44 \\
\hline Daytime SBP & $0.92(0.77-1.09)$ & 0.35 & $1.25(1.04-1.49)$ & 0.01 \\
\hline Daytime DBP & $1.10(1.02-1.20)$ & 0.01 & $1.07(1.00-1.14)$ & 0.04 \\
\hline Nighttime SBP & $0.97(0.94-1.02)$ & 0.30 & $0.99(0.89-1.11)$ & 0.98 \\
\hline Nighttime DBP & $0.95(0.89-1.03)$ & 0.24 & $1.02(0.85-1.22)$ & 0.80 \\
\hline 24-hour SBP & $1.99(0.94-1.06)$ & 0.96 & $0.95(0.81-1.10)$ & 0.52 \\
\hline 24-hour DBP & $1.10(0.99-1.21)$ & 0.06 & $1.12(1.02-1.22)$ & 0.01 \\
\hline
\end{tabular}

Poor outcomes combined with cardiac death, myocardial infarction, stroke and hospitalization. EF: Ejection fraction, AOR: Adjusted odds ratio, CI: Confidence interval, ACEI: Angiotensinconverting enzyme inhibitors, SBP: Systolic blood pressure, DBP: diastolic blood pressure 


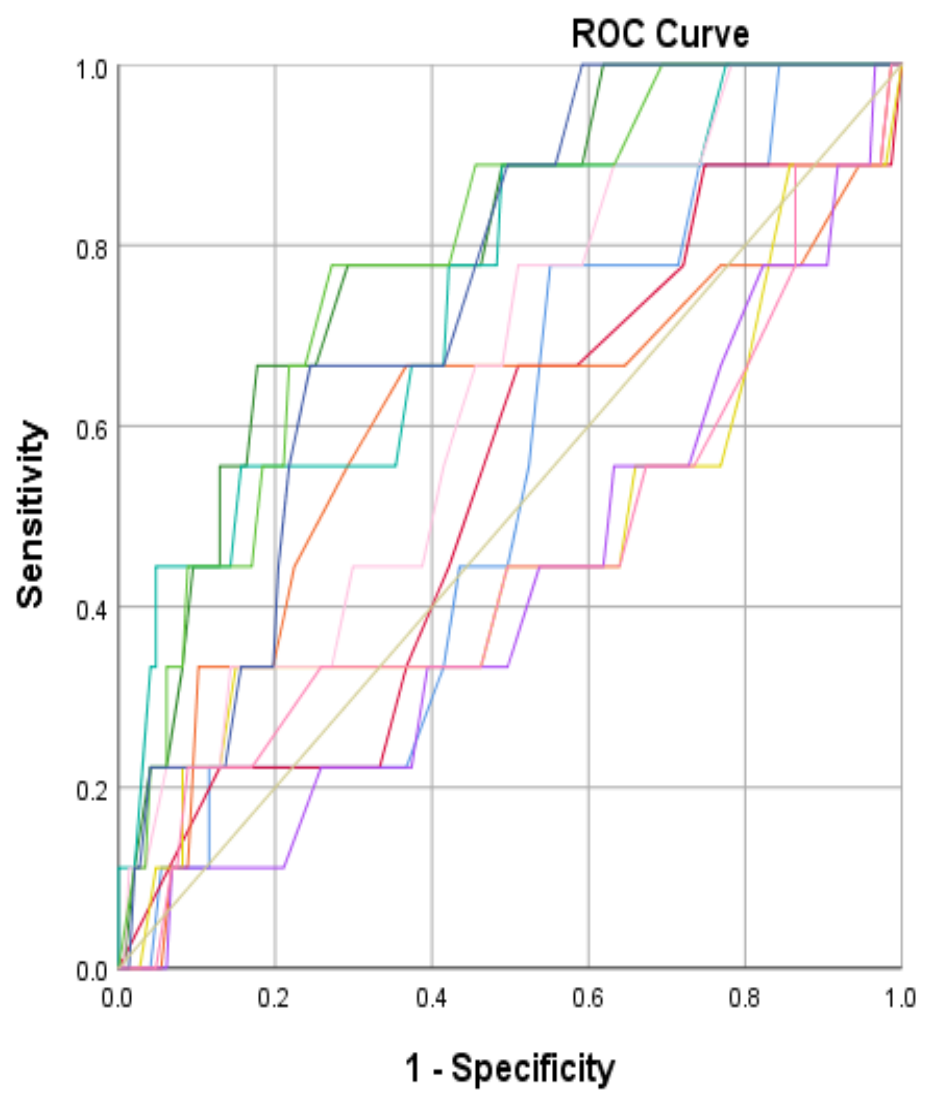

\author{
Source of the \\ Curve \\ - Age \\ -Ejection fraction \\ - Daytime SBP \\ - Daytime DBP \\ Daytime PP \\ - Nighttime SBP \\ Nighttime DBP \\ - Nighttime PP \\ -24-h SBP \\ -24-h DBP \\ - 24-h PP \\ - Reference Line
}

\title{
Diagonal segments are produced by ties.
}

Figure 1: A receiver operating characteristic study determines the prognostic significance of short-term blood pressure variability in patients with endpoints. EF: ejection fraction, daytime SBP: daytime systolic blood pressure, daytime DBP: Daytime diastolic Blood pressure, daytime PP: daytime pulse pressure, night-time SBP: Night-time systolic blood pressure, night-time DBP: Night-time diastolic Blood pressure, night-time PP: night-time pulse pressure, 24-h SBP:24-h systolic blood pressure, 24-h DBP: 24-h diastolic Blood pressure, 24-h PP: 24-h pulse pressure.

\section{Discussion}

The current study sheds significant light on the prognosis impact of short-term BP Variability and its effect on HFrEF patients. This research reports that low ARV of 24-hour systolic BP is related to greater chronic heart failure (HFrEF) patients, regardless of risk markers. Daytime SBP variability, daytime DBP variability, night-time SBP variability24-h SBP and 24-h DBP variability were independently related to negative outcomes of HFrEF patients.

The investigations were undertaken to ascertain the impacts of ambulatory BP fluctuation variability in HF patients with reduced EF (Gibelin, Spillner, Bonnan, \& Chevallier, 2003) (Berry et al., 2016). Gilberl in et al. (Gibelin et al., 2003) discovered that 154 individuals with 


\section{International Journal of Medical Science and Health Research}

Vol. 6, No. 01; 2022

ISSN: $2581-3366$

chronic HF had a worsening prognosis of low BP variability followed up for an average of $565+/ 215$ days. The results were confirmed by Berry et al. (Berry et al., 2016); their study showed that low daytime BP variability was linked to the prognosis in systolic heart failure patients, which was confirmed by large recruitment after following up an average of 4.4 years. Our study confirms the results after assessing the impact of BP variability in 156 subjects followed up for mean of 7.6 months. In our study low BP variability was in the daytime systolic BP, night-time systolic BP, 24-hour systolic BP and 24-hour Diastolic BP. Thus, in contrast to earlier research on the predictive significance of BP variability in hypertension, we have revealed a counterintuitive prognostic effect of blood pressure fluctuation in HF with reduced $\mathrm{EF}$ for the occurrence of cardiac death, myocardial infarction, stroke and hospitalization.

\subsection{Factors influencing BPV in $\mathrm{CHF}$}

Low BPV in subjects with Congestive HF is elaborated. Radaelli et al. (Radaelli et al., 1999) found a change in BPV in nine subjects with congestive HF during vigorous activity, with the variability ranging from $\mathrm{BP} 15$ to $41 \mathrm{mmHg}$ in subjects with congestive $\mathrm{HF}$ and control healthy controls (Cowburn, Cleland, Coats, \& Komajda, 1998). The unsolved question is: What causes the reduction of BPV in CHF processes? Although blood pressure components such as systemic vascular resistance or cardiac output are well defined in CHF patients, the mechanism that causes their fluctuations are incompletely clear.

Intrinsic elements such as baroreflex sensitivity(Lanfranchi \& Somers, 2002), autonomic nervous system integrity (Grassi, Bombelli, Seravalle, Dell'Oro, \& Quarti-Trevano, 2010), circadian hormonal secretion, myocardial contractile reserve, and pre-load volume (Giles, Roffidal, Quiroz, Sander, \& Tresznewsky, 1996) are possible routes that might contribute to BPV. Additionally, the aggregate of reactions to external pressor stimuli, spontaneously and regulatory variations due to central nervous system influences, mechanical forces created by breathing, and impacts of humoral and local vasomotor processes determine daily BP variation involved in BPV in systolic CHF patients (L. J. Mena, Felix, Melgarejo, \& Maestre, 2017).

\subsection{BPV prognostic Paradox}

Over the last decades, many investigations have denoted that the typical risk factors for cardiovascular disease have become favorable prognostic indicators for $\mathrm{CHF}$ patients. In a CHF population, high blood pressure, hypercholesterolemia, and a high BMI are all connected to survival (Berry et al., 2016). Furthermore, BPV is required for survival in various complex physiologic adaptive systems (Lanfranchi \& Somers, 2002); a greater level of BPV worsens the prognosis of patients with hypertension (Chen, 2008), while a low amount worsens the CHF prognosis. On the contrary, another paradigm in CHF and BPV must be regarded as part of the epidemiological reversal of traditional risk factors for CHF (Kalantar-Zadeh, Block, Horwich, \& Fonarow, 2004).

\subsection{Limitation}

Although this was single retrospective cohort research with a short median follow-up, it is impossible to exclude measurement mistakes caused by technical difficulties such as the size of 
the cuff or the position of the cuff displacement, which may occur in certain individuals. The monitoring device records the pressure measurements gathered, which should be done by the calibrator often before and during the study using a mercury sphygmomanometer to make sure that the information acquired is accurate. Prospective studies are required to corroborate these findings.

\section{Conclusion}

Low ARV of 24-hour systolic BP may be linked to the cardiovascular risk indicators daytime systolic BP variability, night-time systolic BP variability, 24-h systolic BP variability, and 24-h diastolic BP variability with $\mathrm{HF}$ with reduced EF patients.

\section{Abbreviations}

ABPM: Ambulatory blood pressure monitoring

AOR: Adjusted odds ratio

AM: Ante meridiem

ARV: average real variability

BMI: Body mass index

BBP: systolic blood pressure

BP: Blood pressure

BPV: Blood pressure variability

CAEI: Angiotensin-converting enzyme inhibitors

CHF: chronic heart failure

CI: Confidence interval

DBP: diastolic blood pressure

EF: Ejection fraction

HF: heart failure

HFrEF: heart failure with reduced ejection fraction

HSARV: High systolic Average real variability

LSARV: Low systolic Average real variability

LUSH: Lanzhou University second hospital

mmHg: Millimeters of mercury

M: Mean

MI: Myocardial infarction

NYHA: New York Heart Association functional class

OR: Odds ratio

PM: Post meridiem

PP: pulse pressure

ROC: receiver operating characteristic curve

SD: standard deviation 


\section{International Journal of Medical Science and Health Research}

Vol. 6, No. 01; 2022

ISSN: $2581-3366$

SPSS: Statistical Package for the Social Sciences

\section{Conflict of Interest}

The author stated that they have no competing interests.

\section{References}

Berry, M., Lairez, O., Fourcade, J., Roncalli, J., Carrié, D., Pathak, A., . . Galinier, M. (2016). Prognostic value of systolic short-term blood pressure variability in systolic heart failure. Clinical hypertension, 22(1), 1-6.

Chen, A. F. J. H. r. (2008). Blood pressure variability reduction and organ protection in hypertension treatment. 31(4), 587-588.

Clement, D. L., De Buyzere, M. L., De Bacquer, D. A., De Leeuw, P. W., Duprez, D. A., Fagard, R. H., . . . Six, R. O. (2003). Prognostic value of ambulatory blood-pressure recordings in patients with treated hypertension. New England Journal of Medicine, 348(24), 24072415.

Coccina, F., Pierdomenico, A. M., Cuccurullo, C., \& Pierdomenico, S. D. (2019). Prognostic value of average real variability of systolic blood pressure in elderly treated hypertensive patients. Blood pressure monitoring, 24(4), 179-184.

Cowburn, P. J., Cleland, J. G. F., Coats, A. J. S., \& Komajda, M. (1998). Risk stratification in chronic heart failure. European heart journal, 19(5), 696-710.

Dadlani, A., Madan, K., \& Sawhney, J. J. I. h. j. (2019). Ambulatory blood pressure monitoring in clinical practice. 71(1), 91-97.

Gibelin, P., Spillner, E., Bonnan, S., \& Chevallier, T. (2003). Non-invasive blood pressure variability in chronic heart failure: characteristics and prognostic value. Archives des maladies du coeur et des vaisseaux, 96(10), 955-962.

Giles, T. D., Roffidal, L., Quiroz, A., Sander, G., \& Tresznewsky, O. (1996). Circadian variation in blood pressure and heart rate in nonhypertensive congestive heart failure. Journal of cardiovascular pharmacology, 28(6), 733-740.

Goyal, D., Macfadyen, R. J., Watson, R. D., \& Lip, G. Y. H. (2005). Ambulatory blood pressure monitoring in heart failure: a systematic review. European journal of heart failure, $7(2)$, 149-156.

Grassi, G., Bombelli, M., Seravalle, G., Dell'Oro, R., \& Quarti-Trevano, F. (2010). Diurnal blood pressure variation and sympathetic activity. Hypertension Research, 33(5), 381385 .

Güder, G. 1., Frantz, S., Bauersachs, J., Allolio, B., Wanner, C., Koller, M. T., . . . Störk, S. (2009). Reverse epidemiology in systolic and nonsystolic heart failure: cumulative prognostic benefit of classical cardiovascular risk factors. Circulation: Heart Failure, 2(6), 563-571. 


\section{International Journal of Medical Science and Health Research}

Vol. 6, No. 01; 2022

ISSN: $2581-3366$

Kalantar-Zadeh, K., Block, G., Horwich, T., \& Fonarow, G. C. (2004). Reverse epidemiology of conventional cardiovascular risk factors in patients with chronic heart failure. Journal of the American College of Cardiology, 43(8), 1439-1444.

Lanfranchi, P. A., \& Somers, V. K. (2002). Arterial baroreflex function and cardiovascular variability: interactions and implications. American Journal of Physiology-Regulatory, Integrative and Comparative Physiology, 283(4), R815-R826.

Mena, L., Pintos, S., Queipo, N. V., Aizpurua, J. A., Maestre, G., \& Sulbaran, T. (2005). A reliable index for the prognostic significance of blood pressure variability. Journal of hypertension, 23(3), 505-511.

Mena, L. J., Felix, V. G., Melgarejo, J. D., \& Maestre, G. E. (2017). 24-Hour blood pressure variability assessed by average real variability: a systematic review and meta-analysis. Journal of the American Heart Association, 6(10), e006895.

Palatini, P., Frigo, G., Bertolo, O., Roman, E., Da Corta, R., \& Winnicki, M. J. B. p. m. (1998). Validation of the A\&D TM-2430 device for ambulatory blood pressure monitoring and evaluation of performance according to subjects' characteristics. 3(4), 255-260.

Parati, G., Ochoa, J. E., Lombardi, C., \& Bilo, G. (2013). Assessment and management of bloodpressure variability. Nature Reviews Cardiology, 10(3), 143-155.

Pierdomenico, S. D., Lapenna, D., Guglielmi, M. D., Antidormi, T., Schiavone, C., Cuccurullo, F., \& Mezzetti, A. (1995). Target organ status and serum lipids in patients with white coat hypertension. Hypertension, 26(5), 801-807.

Radaelli, A., Perlangeli, S., Cerutti, M. C., Mircoli, L., Mori, I., Boselli, L., . . . Signorini, G. (1999). Altered blood pressure variability in patients with congestive heart failure. Journal of hypertension, 17(12), 1905-1910.

Raphael, C. E., Whinnett, Z. I., Davies, J., Fontana, M., Ferenczi, E., Manisty, C. H., ... Francis, D. P. J. H. (2009). Quantifying the paradoxical effect of higher systolic blood pressure on mortality in chronic heart failure. 95(1), 56-62.

Regnault, V., Lagrange, J., Pizard, A., Safar, M. E., Fay, R., Pitt, B., . . . Lacolley, P. (2014). Opposite predictive value of pulse pressure and aortic pulse wave velocity on heart failure with reduced left ventricular ejection fraction: insights from an Eplerenone PostAcute Myocardial Infarction Heart Failure Efficacy and Survival Study (EPHESUS) substudy. Hypertension, 63(1), 105-111. 